Foreword

For reprint orders, please contact: reprints@futuremedicine.com

\title{
Diabetes and its lack of causal biomarkers
}

First draft submitted: 7 September 2016; Accepted for publication: 9 September 2016; Published online: 14 October 2016

Keywords: diabetes $\bullet \mathrm{GLP}-1 \bullet$ insulin

Diabetes treatment has had a dramatic effect on patient outcome and quality of life. Historically, diabetes treatment has gone from complete avoidance of carbohydrates (that killed patients) to insulin replacement (that saved patients). Modern treatment modalities have seen, and will continue to see, novel approaches of treating the disorder, where new organ targets include the gut, liver, immune system, kidney, and so on. Perhaps, as a consequence, the classification also went from insulin- and noninsulin-dependent diabetes to Type 1 and Type 2 (and Type 3). Nevertheless, these boxes are still alarmingly crude and leave a large group of patients unclarified in terms of etiology and pathogenesis. But the dichotomization of diabetes as a disease spectrum largely reflects treatment options rather than a molecular understanding. Thus, the current biomarkers available for further aid and precision in helping patients are few and generally powerless. At best, our biomarkers may help predict death and despair from manifest diabetes, but in reality, the markers should aid and optimize treatment for increasing quality and quantity of life, ultimately allowing intervention even before diabetes precipitates.

Increased glucose concentrations in circulation still constitute the definition of diabetes. Moreover, the most predictive marker of coming diabetes is still largely glucose concentrations in fasting plasma. From a mechanistic point of view, however, this definition and marker effect should be considered a late event in the development of diabetes. As such, glucose measurement will hardly prevent diabetes, but mostly define. A major issue then is what occurs before the increased plasma glucose concentration. To put it in simple words: what is actually the primary hit(s) in Type 2 diabetes; which organs may be the first to tell us about the ongoing pathophysiology? As we realize more and more that the endocrine pancreas is not the only affected organ, we turn to other organs for changes that may reflect early events in diabetes development. And that may also be where meaningful biomarkers of the disease complex may lie. By incorporating them into clinical practice, we may then become able to prevent the disease to develop into manifest diabetes, where current data show that it is hard (although always not impossible) to reverse the processes.

Another major challenge for future biomarkers of diabetes seems to be to differentiate obesity-related organ changes from glucose metabolism. Although this is not pursued eagerly, some of the answers may thus lie with obese people, who do not develop diabetes, rather than in obese patients, who do develop diabetes. Vice versa, comparing slim Type 2 diabetes patients with slim nondiabetes individuals still seems like an appealing strategy. From the latter exercise, some information about intra-abdominal fat deposits has arisen. Yet, no stringent biomarkers have surfaced from this observation, and we still do not know exactly why some

Biomarkers

in Medicine

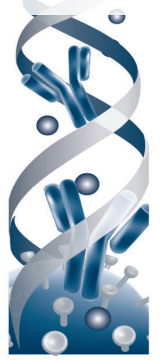

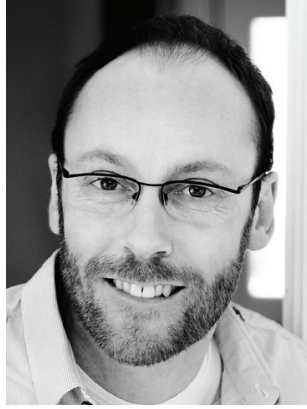

Jens P Goetze

Author for correspondence: Department of Clinical Biochemistry, Rigshospitalet, Copenhagen, Denmark and

Faculty of Health \& Technology, Metropolitan University College, Copenhagen, Denmark Tel.: +4535452202

Fax: +4535452880

JPG@dadlnet.dk

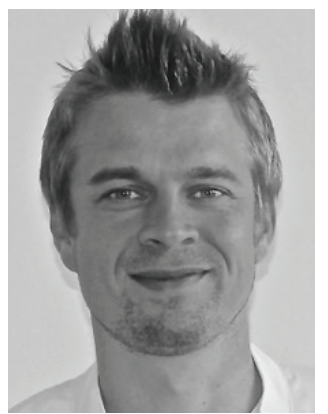

David P Sonne

Center for Diabetes Research, Department of Medicine, Gentofte Hospital, University of Copenhagen, Copenhagen, Denmark 
patients deposit unfortunate fat here, while others do not. Genetic data in Type 2 diabetes have also given us some hints as to pathophysiology. But again, biomarkers that may help us to change development of disease have still not arisen.

\section{"[A] major challenge for future biomarkers of diabetes seems to be to differentiate obesity-related organ changes from glucose metabolism."}

Searching for biomarkers often just follows the current paradigm. If we believe that diabetes is primarily a disorder of the endocrine pancreas, we then tend to look for markers that reflect the endocrine pancreas. If we are to change the field, however, we may need to abandon the current paradigm of diabetes and look elsewhere. One example could be the endocrine heart, where the cardiac expression of natriuretic peptides is decreased. Whether this is a consequence or a prerequisite for diabetes development is not yet clarified, but the event can be readily measured. Another organ that most likely will harbor early biomarkers is the endocrine gut. Glucagon from the endocrine gut seems to be deeply implicated in diabetes pathology - and perhaps more so than pancreatic glucagon homeostasis. Finally, the liver is a major glucoseholding organ. Today, our markers of liver function are mainly restricted to protein synthesis and hepatic necrosis. But the liver does so much more than produce proteins and bile (and metabolize drugs) - its ability to store and release glucose could very well be

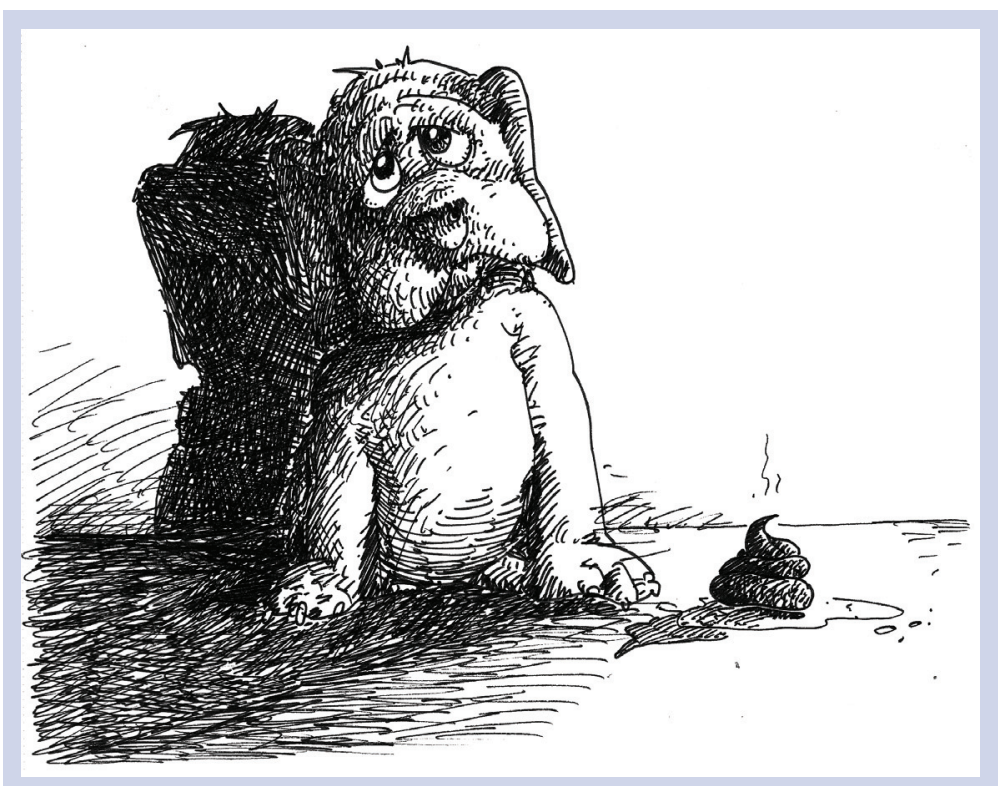

Figure 1. Drawing of a puppy and a poo: A case of causal relation or incidental proximity? Figure courtesy of Senior Biochemist, Jakob Albrethsen (Rigshospitalet, Copenhagen, Denmark). one of the earliest events in diabetes pathology. However, we have no clinical markers of glucose handling within the liver.

In the present issue of Biomarkers of Medicine, selected biomarkers of potential relevance for diabetes assessment is presented. Rehfeld takes us through the incretin effect and reminds us all that the incretin effect was described long before glucagon-like peptide 1 emerged [1]. Hudzik et al. report some original data on the predictive use of a simple score system (sTIPS) for assessing prognosis in diabetes patients having a myocardial infarction [2]. Albrechtsen et al. further focus on glucagon, which may be described as a 'renaissance hormone' in modern diabetes understanding [3]. Krentz and Hompesch run us through the gold standard marker in diabetes, that is, glucose and its laboratory measurement [4]. Notably, basic understanding of glucose measurement is simply key when working with clinical diabetes. Also, the hormonal incretin response is so much more than a glucagon-like peptide 1 story, where gut endocrinology utilizes many peptide hormones for increasing insulin secretion. Horowitz extends the incretin effect and brings in gastric emptying in postprandial glycemic control [5]. Finally, Broedbaek and Hilsted report on chromogranin A; an acidic secretory granule protein that supposedly harbors the inhibitor hormone pancreastatin [6]. However, chromogranin A as a biomarker may also hold some biological information for us concerning overall endocrine secretion.

From the articles presented in this issue, one may speculate on the status of coming biomarkers in diabetes. According to the traditional view of diabetes as a disease of the endocrine pancreas, we feel tempted to regard the pancreas as "guilty as a puppy sitting next to a pile of poo" (quote from the Blackadder). However, much attention to the endocrine pancreas has not given us new biomarkers, where, for instance, characterization of the granular contents in islet cells has not provided us with new molecular markers. So the search must continue with open minds. And it is our gut feeling that the search should not only focus on 'the puppy' but rather its mother. In order to do this, we must still encourage basic research into the pathology of diabetes, which seems the intelligent way of spotting new measures of disease initiation and progression.

In the meantime, it should be recapitulated that biomarkers other than molecular measurements already exist. Although we all are concerned with counting our calorie intake and physical achievements, robust measures of inactivity are already at hand. Although not very positive, one could start by counting the 'time spent in front of the television (TV).' This type of inactivity has been closely correlated to the development of 
insulin resistance, and 'the TV' nowadays also includes time spent in the company with an iPad. Clearly, this type of biomarker has no commercial interest, but it may still be an action field, where we can make a direct impact on the diabetes epidemic of the world. It is as simple as pushing the 'off' button - with a dramatic effect on our health and quality of life. And while in the 'TV/iPad off' mode, we could even take the puppy for a walk (Figure 1).

\section{References}

1 Rehfeld J. Cholecystokinin and gastrin in diabetes mellitus. Biomark. Med. 10(11), 1125-1127 (2016).

2 Hudzik B, SzkodziŃski J, Wasilewski J et al. A novel simplified thrombo-inflammatory score portends poor outcome in diabetic patients following myocardial infarction. Biomark. Med. 10(11), 1129-1139 (2016).

3 Wewer Albrechtsen N, Kuhre R, Pedersen J, Knop F, Holst J. The biology of glucagon and the consequences of hyperglucagonaemia. Biomark. Med. 10(11), 1141-1151 (2016).

\section{Financial \& competing interests disclosure}

The authors have no relevant affiliations or financial involvement with any organization or entity with a financial interest in or financial conflict with the subject matter or materials discussed in the manuscript. This includes employment, consultancies, honoraria, stock ownership or options, expert testimony, grants or patents received or pending, or royalties.

No writing assistance was utilized in the production of this manuscript.

4 Krentz A, Hompesch M. Glucose: archetypal biomarker in diabetes diagnosis, management and research. Biomark. Med. 10(11), 1153-1166 (2016).

5 Wu T, Rayner C, Horowitz M. Inter-regulation of gastric emptying and incretin hormone secretion: implications for postprandial glycaemic control. Biomark. Med. 10(11), 1167-1179 (2016).

6 Broedbaek K, Hilsted L. Chromogranin A as biomarker in diabetes. Biomark. Med. 10(11), 1181-1189 (2016). 